\title{
Rayleigh waves in Cosserat elastic materials
}

\author{
Stan Chiriţă \\ Faculty of Mathematics, Al. I. Cuza University of Iaşi, Blvd. Carol I, no. 11, 700506 - Iaşi, Romania \\ Ionel-Dumitrel Ghiba \\ Faculty of Mathematics, Al. I. Cuza University of Iaşi, Blvd. Carol I, no. 11, 700506 - Iaşi, Romania \\ and "Octav Mayer" Mathematics Institute, The Romanian Academy, Iaşi Branch, Blvd. Carol I, no. 8, \\ 700505 - Iaşi, Romania
}

\begin{abstract}
The present paper gives explicit solutions for surface waves propagation in a homogeneous half space filled with an isotropic Cosserat elastic material. Such solutions are important in the study of seismic waves in an earthquake, supposing that the bottom land is modeled as having a microstructure. To construct explicit expressions for the possible surface waves under consideration, we use the Stroh formalism. These solutions are further used to study the Rayleigh waves and to give the explicit equation for the Rayleigh surface wave speed (secular equation). Numerical calculations and graphics corresponding to the analytical solution are given for aluminium-epoxy composite.
\end{abstract}

Keywords: Cosserat elastic half space, Rayleigh waves, Stroh formalism, Secular equation 2000 MSC: 74J15, 74M25, 74H05, 74J05

\section{Introduction}

This paper is concerned with the seismic waves propagation in Cosserat elastic materials. The theory of elastic materials with microstructure goes back to the book of Cosserat \& Cosserat (1909). After that, the theory of materials with microstructure became a subject of intensive study in literature (see, for example, Toupin (1962); Mindlin (1963, 1964); Eringen \& Suhubi (1964a,b)). Eringen (1966) introduced the concept of micropolar continua, which is similar with Cosserat continua; additionally he introduced a conservation law for the microinertia tensor, as a special case of micromorphic continua (Eringen \& Suhubi, 1964a,b).

It is well-known that the response of the material to external stimuli depends heavily on the motions of its inner structure. Classical elasticity ignores this effect by ascribing

Email addresses: schirita@uaic.ro (Stan Chiriţă), ghiba_dumitrel@yahoo.com (Ionel-Dumitrel Ghiba) 
only translation degrees of freedom to material points of the body. In the micropolar continuum theory, the rotational degrees of freedom play a central role. Thus, we have six degrees of freedom, instead of the three ones considered in classical elasticity. Moreover, in micropolar theories, in order to characterize the force applied on the surface element, two tensors are used: an asymmetric stress tensor and a couple stress tensor. Crystals, composites, polymers, suspensions, blood, grid and multibar systems can be considered as examples of media with microstructure. In fact, nature abounds with many substances which point out the necessity for the considering of micromotions into the mechanical studies. A review of the historical developments as well as references to various contributions on the subject may be found in the monographs by Truesdell \& Noll (1965), Nowacki (1986), Eringen (1999) and Ieşan (2004).

The classical theory of elasticity does not explain certain discrepancies that occur in the case of problems involving elastic vibrations of high frequency and short wavelength, that is, vibrations generated by ultrasonic waves. According to the book of Eringen (1999), if the ratio of the characteristic length associated with the external stimuli and the internal characteristic length is in the neighborhood of 1 , then the response of constituent subcontinua becomes important. This is the reason why the short wavelenght behavior departs drastically from experimental observations in classical elasticity. The micropolar effects become important in high-frequency and short wave-length regions of waves.

We have to outline that Kulesh et al. (2005, 2006) have studied the propagation of elastic surface waves in Cosserat medium and have sought the solutions in the form of wave packets determined by an arbitrary-shape Fourier spectrum. So, the solution is given in the form of Fourier integrals. In some previous papers (see, for example, (Eringen, 1999) and the papers cited therein) the authors have considered some lower bounds for the frequency and also for the wave-number; also, the attenuating coefficient and some conditions upon wave speed and upon wave-number are not explicitly given in the previous papers (see (Kulesh et al., 2006; Erofeyev, 2003)). These are consequences of the methods used in their approaches. For the gradient type approach of microstructured solids the propagation of surface waves was studied by (Georgiadis \& Velgaki, 2003; Georgiadis et al., 2004). Moreover, the class for which the generalized form of the secular equation has an admissible solution does not established yet.

Our main purpose is to construct new solutions for the waves propagation problem in a micropolar half space. In fact, we use the Stroh formalism (Stroh, 1962; Destrade, 2007) in order to obtain explicit expressions for the possible surface waves in concern and, moreover, we obtain a sextic equation with real coefficients for the propagation condition. Further, we give explicit expressions of the attenuating coefficients and explicit conditions upon wave speed. After that we give the exact expressions of three linear independent amplitude vectors. These amplitudes are characteristic for the coupled case of the elastic and microstructure effects. In the last part of the paper, these inhomogeneous plane wave solutions are used to study the Rayleigh surface waves (Rayleigh, 1885) in an isotropic Cosserat elastic half space. An explicit equation is also established for the Rayleigh wave speed (secular equation). This equation has a simple form, it is not a generalization of the secular equation from the classical elasticity and it is a special one valid for the genuine micropolar model. Moreover, by comparing it with other generalized forms of the secular 
equation (Eringen, 1999; Kulesh et al., 2006; Erofeyev, 2003; Koebke \& Weitsman, 1971) (see, also, (Chiriţă \& Ghiba, 2010)) this equation does not involve the complex form of the attenuating coefficients, and for this reason we consider that it has to be most appropriate for considerations in further studies. For a specified class of materials we prove that this equation always has at least one admissible solution. The conditions imposed upon the constitutive quantities are in concordance with those expressed by the positive definiteness of the internal energy and include the materials considered by Gauthier \& Jahsman (1975) and Gauthier (1982) (see also (Eringen, 1999), Sections 5.11-5.13) in their experiments. In fact, from the illustrative graphics which we give within this paper one can see that it is possible to have only one admissible wave speed for each material. To obtain such solutions we do not impose any lower bounds to the frequency or to the wave-number. Moreover, the Rayleigh wave solution is also valid for a complementary class of materials with respect to those previously considered in the literature.

We have to notice that the Rayleigh wave problem in the classical theory of linear elasticity has been a subject of great interest in literature on the field (see, for example, the article by Hayes \& Rivlin (1962) and the monographs by Jeffreys (1952) and Achenbach (1973)). Important contributions on this argument have been reported recently by Rahman \& Barber (1995), Nkemzi (1997, 2008), Malischewsky (2000), Vinh \& Ogden (2004), Li (2006), Destrade (2007), Vinh \& Malischewsky (2007), Vinh \& Malischewsky (2008) and Ting (2011a,b).

\section{Basic equations of the Cosserat elastic model}

Throughout this section $B$ is a bounded regular region of three-dimensional Euclidean space. We let $\partial B$ denote the boundary on $B$, and designate by $\mathbf{n}$ the outward unit normal on $\partial B$. We assume that the body occupying $B$ is a linearly Cosserat elastic material. The body is referred to a fixed system of rectangular Cartesian axes $O x_{i}(i=1,2,3)$. Throughout this paper Latin indices have the range 1, 2, 3, Greek indices have the range 1, 2 and the usual summation convention is employed. We use a subscript preceded by

a comma for partial differentiation with respect to the corresponding coordinate and a superposed dot represents the derivative with respect to time variable.

The deformation of such a medium is described by two fields, namely, the displacement field $\mathbf{u}$ and the microrotation field $\varphi$ over $B \times[0, \infty)$.

Within the framework of the linear theory, the constitutive equations for a homogeneous and isotropic Cosserat elastic solid are (Eringen, 1999; Ieşan, 2004)

$$
\begin{aligned}
t_{i j} & =\lambda e_{r r} \delta_{i j}+(\mu+\kappa) e_{i j}+\mu e_{j i}, \\
m_{i j} & =\alpha \varkappa_{r r} \delta_{i j}+\beta \varkappa_{j i}+\gamma \varkappa_{i j},
\end{aligned}
$$

where $t_{i j}$ are the components of the stress tensor, $m_{i j}$ are the components of the couple stress tensor, $\lambda, \mu, \kappa, \alpha, \beta$ and $\gamma$ are elastic moduli, $\delta_{i j}$ is the Kronecker delta and the strain measures $e_{i j}$ and $\varkappa_{i j}$ are given by the geometrical equations

$$
e_{i j}=u_{j, i}+\varepsilon_{j i k} \varphi_{k}, \quad \varkappa_{i j}=\varphi_{j, i}
$$


$u_{i}$ are the components of the displacement vector and $\varphi_{i}$ are the components of the microrotation vector.

In the absence of the body force and the body couple, the equations of motion of the linear theory of Cosserat elastic materials are given by (Eringen, 1999; Ieşan, 2004)

$$
\begin{aligned}
t_{j i, j} & =\varrho \ddot{u}_{i}, \\
m_{j i, j}+\varepsilon_{i r s} t_{r s} & =\varrho j \ddot{\varphi}_{i},
\end{aligned}
$$

where $\varrho$ is the density mass and $j$ is the inertia coefficient.

The surface force and the surface couple at a regular point $\mathbf{x}$ of the boundary $\partial B$ are given by

$$
t_{i}=t_{j i} n_{j}, \quad m_{i}=m_{j i} n_{j}
$$

where $n_{j}=\cos \left(\mathbf{n}_{\mathbf{x}}, O x_{j}\right)$ and $\mathbf{n}_{\mathbf{x}}$ is the unit vector of the outward normal to $\partial B$ at $\mathbf{x}$.

In the case of isotropic linear micropolar elastic materials, the internal energy $W$ per unit volume is given by

$$
2 W(\mathbf{e}, \varkappa)=\lambda e_{r r} e_{s s}+(\mu+\kappa) e_{i j} e_{i j}+\mu e_{i j} e_{j i}+\alpha \varkappa_{r r} \varkappa_{s s}+\beta \varkappa_{j i} \varkappa_{i j}+\gamma \varkappa_{i j} \varkappa_{i j} .
$$

The necessary and sufficient conditions for the internal energy density $W$ to be a positive definite quadratic form in terms of $e_{i j}$ and $\varkappa_{i j}$ are

$$
\begin{gathered}
3 \lambda+2 \mu+\kappa>0, \quad 2 \mu+\kappa>0, \quad \kappa>0, \\
3 \alpha+\beta+\gamma>0, \quad \gamma+\beta>0, \quad \gamma-\beta>0 .
\end{gathered}
$$

Further, we also suppose that $\varrho$ and $J=\varrho j$ are strictly positive numbers. Our analysis is within this framework.

In the present paper, we shall consider the region $B$ to be the half space $\Sigma: x_{2} \geq 0$ subject to a process of plane strain parallel to the $x_{1} O x_{2}$ plane, that is

$$
\begin{aligned}
& u_{\beta}=u_{\beta}\left(x_{1}, x_{2}, t\right), \quad u_{3}=0, \\
& \varphi_{\beta}=0, \quad \varphi_{3}=\varphi\left(x_{1}, x_{2}, t\right), \quad\left(x_{1}, x_{2}, x_{3}\right) \in \Sigma, \quad t \geq 0 .
\end{aligned}
$$

So, all the quantities defined above are independent of $x_{3}$, and we have the relevant non-zero strain measures

$$
e_{\alpha \beta}=u_{\beta, \alpha}+\varepsilon_{\beta \alpha 3} \varphi, \quad \varkappa_{\alpha 3}=\varphi_{, \alpha},
$$

while the equations of motion (2.3) are reduced to

$$
\begin{aligned}
t_{\beta \alpha, \beta} & =\varrho \ddot{u}_{\alpha}, \\
m_{\beta 3, \beta}+t_{12}-t_{21} & =J \ddot{\varphi},
\end{aligned}
$$

where

$$
\begin{aligned}
t_{\alpha \beta} & =\lambda e_{\varrho \varrho} \delta_{\alpha \beta}+(\mu+\kappa) e_{\alpha \beta}+\mu e_{\beta \alpha}, \\
m_{\varrho 3} & =\gamma \varkappa_{\varrho 3} .
\end{aligned}
$$




\section{Stroh formalism for plane waves}

We will treat the system of partial differential equations $\mathcal{S}$ defined by (2.8), (2.9) and (2.10) in the considered homogeneous micropolar elastic half space by means of the plane waves. Without loss in generality we will study the waves propagating along the $x_{1}$-axis with speed $v$ and wave-number $k$ in the $x_{1}$-direction. Thus, we seek solutions of the above half space problem of the form

$$
\left\{u_{1}, u_{2}, \varphi\right\}\left(x_{1}, x_{2}, t\right)=\left\{U_{1}, U_{2}, \phi\right\}\left(x_{2}\right) e^{\mathrm{i} k\left(x_{1}-v t\right)},
$$

and

$$
\left\{t_{11}, t_{22}, t_{12}, t_{21}, m_{13}, m_{23}\right\}\left(x_{1}, x_{2}, t\right)=\mathrm{i}\left\{T_{11}, T_{22}, T_{12}, T_{21}, M_{13}, M_{23}\right\}\left(x_{2}\right) e^{\mathrm{i} k\left(x_{1}-v t\right)},
$$

where $\mathrm{i}=\sqrt{-1}$ is the unit complex.

The system $\mathcal{S}$ lead to a first-order differential system

$$
\mathcal{U}^{\prime}\left(x_{2}\right)=\mathrm{i} k \mathcal{N} \mathcal{U}\left(x_{2}\right),
$$

for

$$
\mathcal{U}\left(x_{2}\right)=\left\{U_{1}, U_{2}, \phi, T_{21}, T_{22}, M_{23}\right\}^{T}\left(x_{2}\right)
$$

where

$$
\begin{gathered}
\mathcal{N}=\left(\begin{array}{cc}
\mathcal{N}_{1} & \mathcal{N}_{2} \\
\mathcal{N}_{3} & \overline{\mathcal{N}}_{1}^{T}
\end{array}\right), \\
\mathcal{N}_{1}=\left(\begin{array}{ccc}
0 & -1+\frac{\kappa}{c_{66}} & \frac{\mathrm{i} \kappa}{k c_{66}} \\
-\frac{c_{12}}{c_{11}} & 0 & 0 \\
0 & 0 & 0
\end{array}\right), \\
\mathcal{N}_{2}=\left(\begin{array}{ccc}
\frac{1}{k c_{66}} & 0 & 0 \\
0 & \frac{1}{k c_{11}} & 0 \\
0 & 0 & \frac{1}{k \gamma}
\end{array}\right), \\
\mathcal{N}_{3}=\left(\begin{array}{ccc}
-k\left(c^{0}-\varrho v^{2}\right) \quad & -\mathrm{i} \kappa\left(2-\frac{\kappa}{c_{66}}\right) \\
0 & \mathrm{i} \kappa\left(2-\frac{\kappa}{c_{66}}\right) & -\gamma k-\frac{\kappa}{k}\left(2-\frac{\kappa}{c_{66}}\right)+J v^{2} k
\end{array}\right),
\end{gathered}
$$

and

$$
c_{11}=\lambda+2 \mu+\kappa, \quad c_{12}=\lambda, \quad c_{66}=\mu+\kappa, \quad c^{0}=c_{11}-\frac{c_{12}^{2}}{c_{11}},
$$


Here, $\overline{\mathcal{N}}_{1}^{T}$ denotes the transposed matrix of the complex conjugate of the matrix $\mathcal{N}_{1}$.

Moreover, we require that the solutions to be attenuated in the direction $x_{2}$, so that they are decaying with distance from the plane surface $x_{2}=0$, that is we require that

$$
\lim _{x_{2} \rightarrow \infty}\left\{u_{1}, u_{2}, \varphi, t_{11}, t_{22}, t_{12}, t_{21}, m_{13}, m_{23}\right\}\left(x_{1}, x_{2}, t\right)=0,
$$

for all $x_{1} \in \mathbb{R}$ and $t \in[0, \infty)$.

Thus, we seek solutions of the above differential system in the form

$$
\mathcal{U}\left(x_{2}\right)=\mathcal{V} e^{i k r x_{2}},
$$

with

$$
\operatorname{Im}(r)>0,
$$

where $\mathcal{V}=\left\{v_{1}, v_{2}, \chi, S_{21}, S_{22}, N_{23}\right\}^{T}$ is a constant vector and $r$ is a complex parameter. Therefore, $\mathcal{V}$ and $r$ satisfy the following eigenvalue problem

$$
\mathcal{N} \mathcal{V}=r \mathcal{V}
$$

The inequality (3.12) ensures that (3.10) holds true.

The characteristic equation corresponding to the above eigenvalue problem is

$$
r^{6}+P_{1} r^{4}+P_{2} r^{2}+P_{3}=0
$$

where

$$
\begin{aligned}
& P_{1}=C_{1}+C_{2}+C_{3}, \\
& P_{2}=C_{1} C_{2}+C_{2} C_{3}+C_{3} C_{1}-\frac{\varrho \kappa^{2} v^{2}}{\gamma k^{2} c_{66}^{2}}, \\
& P_{3}=C_{1} C_{2} C_{3}-\frac{\varrho \kappa^{2} v^{2}}{\gamma k^{2} c_{66}^{2}} C_{1}, \\
& C_{1}=1-\frac{\varrho v^{2}}{c_{11}}, C_{2}=1-\frac{\varrho v^{2}}{c_{66}} \\
& C_{3}=1+\frac{\kappa\left(2 c_{66}-\kappa\right)}{\gamma k^{2} c_{66}}-\frac{J v^{2}}{\gamma} .
\end{aligned}
$$

It is easy to see that the characteristic equation can be written in the following form

$$
\left(r^{2}+C_{1}\right)\left[\left(r^{2}+C_{2}\right)\left(r^{2}+C_{3}\right)-\frac{\varrho \kappa^{2} v^{2}}{\gamma k^{2} c_{66}^{2}}\right]=0,
$$

whose solutions satisfy

$$
\begin{aligned}
& r_{1}^{2}=-C_{1}, \\
& r_{2,3}^{2}=\frac{1}{2}\left[-\left(C_{2}+C_{3}\right) \pm \sqrt{\left(C_{2}-C_{3}\right)^{2}+\frac{4 \varrho \kappa^{2} v^{2}}{\gamma k^{2} c_{66}^{2}}}\right] .
\end{aligned}
$$


The above quantities are admissible roots if and only if the inequality (3.12) is satisfied. So, we must have

$$
C_{1}>0, \quad C_{2}+C_{3}>0, \quad C_{2} C_{3}>\frac{\varrho \kappa^{2} v^{2}}{\gamma k^{2} c_{66}^{2}} .
$$

These conditions are equivalent with

$$
C_{1}>0, \quad C_{2}>0, \quad C_{3}>0, \quad C_{2} C_{3}>\frac{\varrho \kappa^{2} v^{2}}{\gamma k^{2} c_{66}^{2}},
$$

and further they are satisfied if and only if the wave speed $v$ is so that

$$
v^{2}<\min \left\{\mathfrak{c}_{1}, \mathfrak{c}_{2}, \mathfrak{c}_{3}, \mathfrak{c}_{4}\right\}
$$

or

$$
\mathfrak{c}_{5}<v^{2}<\min \left\{\mathfrak{c}_{1}, \mathfrak{c}_{2}, \mathfrak{c}_{3}\right\}
$$

where

$$
\begin{gathered}
\mathfrak{c}_{1}=\frac{c_{11}}{\varrho}, \\
\mathfrak{c}_{2}=\frac{c_{66}}{\varrho}, \\
\mathfrak{c}_{3}=\frac{\gamma}{J}\left[1+\frac{\kappa\left(2 c_{66}-\kappa\right)}{\gamma k^{2} c_{66}}\right] \\
\mathfrak{c}_{4}=\frac{1}{2}\left(\mathfrak{c}_{2}+\mathfrak{c}_{3}+\frac{\varkappa^{2}}{k^{2} J c_{66}}-\sqrt{\Delta}\right), \\
\mathfrak{c}_{5}=\frac{1}{2}\left(\mathfrak{c}_{2}+\mathfrak{c}_{3}+\frac{\varkappa^{2}}{k^{2} J c_{66}}+\sqrt{\Delta}\right),
\end{gathered}
$$

and

$$
\Delta=\left(\mathfrak{c}_{2}-\mathfrak{c}_{3}\right)^{2}+2\left(\mathfrak{c}_{2}+\mathfrak{c}_{3}\right) \frac{\varkappa^{2}}{k^{2} J c_{66}}+\left(\frac{\varkappa^{2}}{k^{2} J c_{66}}\right)^{2}>0 .
$$

Furthermore, it follows from the relations (3.22)-(3.25) that

$$
\mathfrak{c}_{4}<\mathfrak{c}_{2}<\mathfrak{c}_{5}
$$

and hence the possibility that (3.21) be true is avoided. Moreover, we have

$$
\mathfrak{c}_{3}-\mathfrak{c}_{4}=\frac{1}{2}\left(\sqrt{\left(\mathfrak{c}_{2}-\mathfrak{c}_{3}\right)^{2}+\left(\frac{\varkappa^{2}}{k^{2} J c_{66}}\right)^{2}+2\left(\mathfrak{c}_{2}+\mathfrak{c}_{3}\right) \frac{\varkappa^{2}}{k^{2} J c_{66}}}-\left(\mathfrak{c}_{2}-\mathfrak{c}_{3}\right)-\frac{\varkappa^{2}}{k^{2} J c_{66}}\right)>0
$$

and, therefore, we obtain

$$
\mathfrak{c}_{3}>\mathfrak{c}_{4}
$$

Concluding, from (3.20), (3.26) and (3.28), we deduce that the wave speed $v$ is admissible if

$$
v^{2}<\mathfrak{c} \equiv \min \left\{\mathfrak{c}_{1}, \mathfrak{c}_{4}\right\}
$$


In the present paper we consider a homogeneous and isotropic body and so we have explicit expressions for the coefficients of the sextic equation. So we can obtain the eigenvalue $r$ and we can find explicit conditions upon the wave speed $v$ by direct calculation. However, we have to mention that for more complex situations (as, for example, an inhomogeneous or anisotropic body), the method described in (Ting, 2011a,b) can be used to prove that the propagation condition has real coefficients. Moreover, if the case of exponentially graded materials is considered, the impedance matrix method of Barnett and Lothe (Ting, 2011a,b) is highly recommended.

Corresponding to the eigenvalue $r_{1}$ of the matrix $\mathcal{A}$, we find the eigenvector

$$
\begin{aligned}
v_{1}^{(1)} & =\frac{1}{k}, \\
v_{2}^{(1)} & =\frac{1}{k} r_{1}, \\
\chi^{(1)} & =0, \\
S_{21}^{(1)} & =\left(c_{11}-c_{12}\right) r_{1}, \\
S_{22}^{(1)} & =c_{11} r_{1}^{2}+c_{12}, \\
N_{23}^{(1)} & =0,
\end{aligned}
$$

while, corresponding to the wave speeds $r_{2,3}$, we find the following sets of eigenvectors

$$
\begin{aligned}
v_{1}^{(2,3)} & =\frac{\kappa}{c_{66} k} r_{2,3}, \\
v_{2}^{(2,3)} & =-\frac{\kappa}{c_{66} k}, \\
\chi^{(2,3)} & =-\mathrm{i}\left(r_{2,3}^{2}+C_{2}\right), \\
S_{21}^{(2,3)} & =\frac{\kappa}{c_{66}}\left(c_{12}-C_{1} c_{11}\right), \\
S_{22}^{(2,3)} & =\frac{\kappa}{c_{66}}\left(c_{12}-c_{11}\right) r_{2,3}, \\
N_{23}^{(2,3)} & =-\mathrm{i} k \gamma r_{2,3}\left(r_{2,3}^{2}+C_{2}\right) .
\end{aligned}
$$

\section{Rayleigh waves}

In this section we consider that the Cosserat elastic material occupying the half space $x_{2} \geq 0$ is homogeneous and isotropic and we consider Rayleigh waves on the Earth's surface. We assume that the body force and the body couple are absent and the boundary $x_{2}=0$ is traction free and couple traction free, that is, we assume

$$
\begin{aligned}
& t_{21}\left(x_{1}, 0, x_{3}, t\right)=0, \quad t_{22}\left(x_{1}, 0, x_{3}, t\right)=0, \\
& m_{23}\left(x_{1}, 0, x_{3}, t\right)=0 \text { for all } x_{1}, x_{3} \in \mathbb{R}, t \in[0, \infty) .
\end{aligned}
$$

Moreover, we require that the solutions be attenuated in the direction $x_{2}$, so that they are decaying with the distance from the plane surface, that is we require that (3.10) holds true. 
Without loss of generality we will study the waves propagating along the $x_{1}$-axis. We will treat the above problem by means of the inhomogeneous plane waves as developed in the previous section. Thus, we seek solutions of the above free half space problem as a linear combination of the eigenvectors $\mathcal{V}^{(j)}=\left\{v_{1}^{(j)}, v_{2}^{(j)}, \chi^{(j)}, S_{21}^{(j)}, S_{22}^{(j)}, N_{23}^{(j)}\right\}^{T}, j=1,2,3$, that is we seek a solution in the following form

$$
\mathcal{V}=a_{1} \mathcal{V}^{(1)}+a_{2} \mathcal{V}^{(2)}+a_{3} \mathcal{V}^{(3)}
$$

where $a_{1}, a_{2}$ and $a_{3}$ are parameters which will be determined in order that the boundary conditions (4.1) to be satisfied. More exactly, these unknown parameters are solutions of the following algebraic system

$$
\begin{aligned}
& S_{21}=a_{1} S_{21}^{(1)}+a_{2} S_{21}^{(2)}+a_{3} S_{21}^{(3)}=0, \\
& S_{22}=a_{1} S_{22}^{(1)}+a_{2} S_{22}^{(2)}+a_{3} S_{22}^{(3)}=0, \\
& N_{23}=a_{1} N_{23}^{(1)}+a_{2} N_{23}^{(2)}+a_{3} N_{23}^{(3)}=0 .
\end{aligned}
$$

This homogeneous algebraic linear system has non-trivial solutions, if and only if

$$
\Delta_{0}=\left|\begin{array}{ccc}
\left(c_{11}-c_{12}\right) r_{1} & \kappa\left(c_{12}-c_{11} C_{1}\right) & \kappa\left(c_{12}-c_{11} C_{1}\right) \\
c_{11} r_{1}^{2}+c_{12} & \kappa\left(c_{12}-c_{11}\right) r_{2} & \kappa\left(c_{12}-c_{11}\right) r_{3} \\
0 & -\mathrm{i} k \gamma c_{66} r_{2}\left(r_{2}^{2}+C_{2}\right) & -\mathrm{i} k \gamma c_{66} r_{3}\left(r_{3}^{2}+C_{2}\right)
\end{array}\right|=0
$$

On the other hand, in view of the condition (3.29), the roots $r_{s}, s=1,2,3$, can not be equal. So, the relation (4.4) implies

$$
r_{1} r_{2} r_{3}\left(r_{2}+r_{3}\right)\left(c_{11}-c_{12}\right)^{2}+\left(r_{2}^{2}+r_{3}^{2}+r_{2} r_{3}+C_{2}\right)\left(c_{12}-c_{11} C_{1}\right)^{2}=0
$$

In view of (3.12) and (3.14)-(3.19), we have that

$$
\begin{aligned}
& r_{1}=\mathrm{i} \sqrt{C_{1}}, \\
& r_{2} r_{3}=-\sqrt{P_{2}-C_{1} C_{2}-C_{1} C_{3}}, \\
& r_{2}^{2}+r_{3}^{2}=-\left(C_{2}+C_{3}\right), \\
& r_{2}+r_{3}=\mathrm{i} \sqrt{C_{2}+C_{3}+2 \sqrt{P_{2}-C_{1} C_{2}-C_{1} C_{3}}}, \\
& r_{1} r_{2} r_{3}=-\mathrm{i} \sqrt{P_{3}} .
\end{aligned}
$$

It follows that the system (4.3) has a non-trivial solution if and only if the wave speed $v$ satisfies the inequality (3.29) and the following equation

$$
\begin{aligned}
R(v) \equiv & \sqrt{P_{3}(v)\left(C_{2}(v)+C_{3}(v)+2 \sqrt{P(v)}\right)}\left(c_{11}-c_{12}\right)^{2}- \\
& -\left(C_{3}(v)+\sqrt{P(v)}\right)\left(c_{12}-c_{11} C_{1}(v)\right)^{2}=0
\end{aligned}
$$

where

$$
P(v)=P_{2}(v)-C_{1}(v) C_{2}(v)-C_{1}(v) C_{3}(v)
$$


In the Section 5 we exemplify the above analysis with some numerical results relating the materials considered by Gauthier \& Jahsman (1975) and Gauthier (1982) in their experiments. For the quantities which they have considered there and for $k=1$, the above function has the graphic given by Figure 1. This graphic proves that for the respective micropolar elastic material the wave speed is $v \approx 0.873217$.

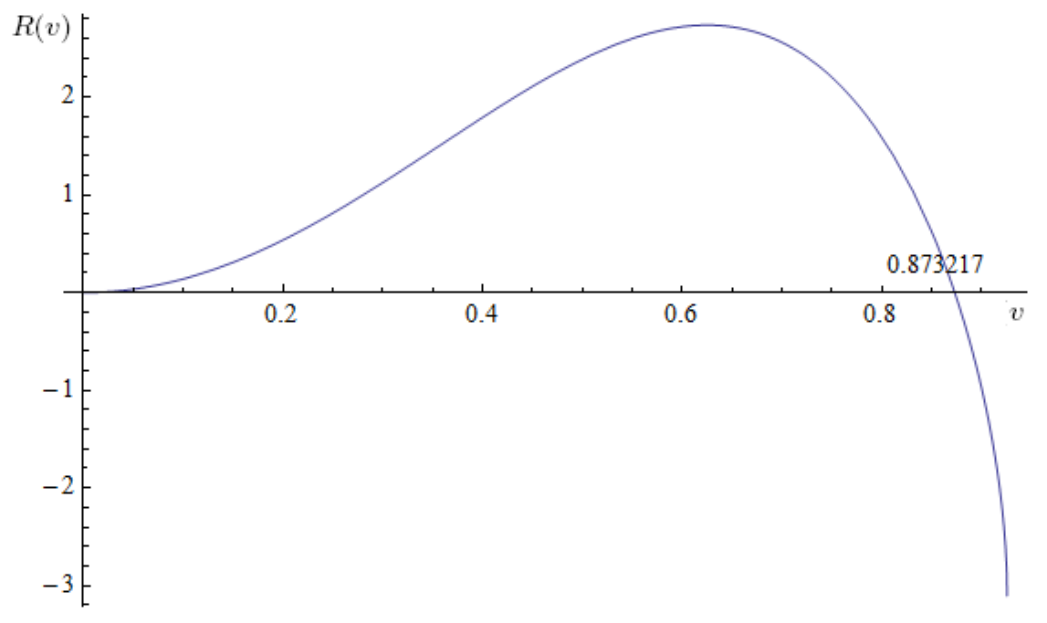

1: The graphic of $R(v)$ on the interval $[0,0.925689)$.

Further, inspired by this graphic, we prove that the secular equation (4.7) has at least a solution for each isotropic micropolar elastic material having a positive definite internal energy. To this end we first note that

$$
\lim _{v \rightarrow \sqrt{c_{1}}} P_{3}(v)=0, \quad \lim _{v \rightarrow \sqrt{c_{4}}} P_{3}(v)=0,
$$

and further, we conclude from (4.7) that

$$
\lim _{v \rightarrow \sqrt{\mathfrak{c}_{1}}} R(v)<0, \quad \lim _{v \rightarrow \sqrt{\mathfrak{c}_{4}}} R(v)<0 .
$$

On the other hand, we have to remark that

$$
R(0)=0 .
$$

Let us now consider the function

$$
\begin{aligned}
Q(v) \equiv & R(v)\left[\sqrt{P_{3}(v)\left(C_{3}(v)+C_{2}(v)+2 \sqrt{P(v)}\right)}\left(c_{11}-c_{12}\right)^{2}+\right. \\
& \left.+\left(C_{3}(v)+\sqrt{P(v)}\right)\left(c_{12}-c_{11} C_{1}(v)\right)^{2}\right] .
\end{aligned}
$$

In view of the relation (4.11) we deduce that $Q(0)=0$. Moreover, if we develop $Q(\varepsilon)$, 
with $\varepsilon$ a very small parameter, then we find

$$
\begin{aligned}
& Q(\varepsilon)=\frac{\varrho\left(\kappa-2 c_{66}\right)^{3}}{k^{4} \gamma^{2} c_{11} c_{66}^{2}}\left\{[ \kappa ( 2 c _ { 6 6 } - \kappa ) + k ^ { 2 } \gamma c _ { 6 6 } ] \left[\left(2 c_{66}-\kappa\right)\left(\kappa\left(2 c_{66}-\kappa\right)+2 k^{2} \gamma c_{66}\right)-\right.\right. \\
& \left.-2 c_{11}\left(\kappa\left(2 c_{66}-\kappa\right)+k^{2} \gamma\left(\kappa+2 c_{66}\right)\right)\right]+ \\
& +2 k^{2} \gamma c_{66}\left[-c_{11}\left(2 \kappa\left(2 c_{66}-\kappa\right)+k^{2} \gamma\left(\kappa+2 c_{66}\right)\right)+\right. \\
& \left.\left.+\left(2 c_{66}-\kappa\right)\left(\kappa\left(2 c_{66}-\kappa\right)+k^{2} \gamma c_{66}\right)\right] \sqrt{C_{3}(0)}\right\} \varepsilon^{2}+O\left(\varepsilon^{3}\right) .
\end{aligned}
$$

It is easy to see that, if

$$
\lambda+\mu>0
$$

then, $Q(\varepsilon)$ is a positive quantity for any $\varepsilon>0$ sufficiently small. Let $\varepsilon_{0}>0$ be so that $Q\left(\varepsilon_{0}\right)>0$. Then, for materials characterized by the inequality (4.14), we have

$$
R\left(\varepsilon_{0}\right)>0
$$

On the other hand, in this case, we have

$$
\mathfrak{c}_{4}<\mathfrak{c}_{1}
$$

The function $R$ is continuous on $\left(\varepsilon_{0}, \sqrt{\mathfrak{c}_{4}}\right)$ and hence, in view of the relations $(4.10)$ and (4.15), we can conclude that there is at least one admissible solution of the equation (4.7) in the interval $\left(\varepsilon_{0}, \sqrt{\mathfrak{c}_{4}}\right)$.

We have to outline that, in the classical elasticity, the solution of the corresponding equation for Rayleigh surface waves speed (the secular equation) has been studied numerically by Rayleigh (1885). Jeffreys (1952) and Hayes \& Rivlin (1962) also have studied the secular equation (see also the recent works by Rahman \& Barber (1995), Nkemzi (1997, 2008), Malischewsky (2000), Vinh \& Ogden (2004), Li (2006), Destrade (2007), Vinh \& Malischewsky (2007), Vinh \& Malischewsky (2008) and Ting (2011a,b)). The secular equation (4.7) also poses difficulties and the determination of the exact numerical solutions can be obtained as in the next section. Because we have the exact analytical solutions, the errosrs which can appear in the calculus become very small. But, by comparing with other generalized form of the secular equation ((Eringen, 1999), page 180) (see, also, (Chiriţă \& Ghiba, 2010)), this equation does not involve the complicated form of the attenuating coefficients, and we consider that it will be more appropriate for considerations in further studies.

\section{Some numerical applications for experimental data}

In this section we give numerical computations and some representations for the quantities obtained in the previous sections associated with the aluminium-epoxy composite considered by Gauthier (1982) (see also (Eringen, 1999)). For such a material we have

$$
\begin{gathered}
\lambda=7,59 \mathrm{GPa}, \quad \mu=1,89 \mathrm{GPa}, \frac{\kappa}{\mu}=0,00788 \\
\frac{\kappa}{\rho}=0,0067 \mathrm{~mm}(\mu \mathrm{s})^{-1}, \quad j=0,0196 \mathrm{~mm}^{2}, \frac{\gamma}{\mu j}=7,11 .
\end{gathered}
$$


In what follows, we give first of all the table of values for the wave speed $v$, solution of the secular equation (4.7), for different values of the wave number $k$ and we identify in each case the value of the upper bound, $\sqrt{\mathfrak{c}_{4}}$. 
Table 1. Surface wave speed

\begin{tabular}{|l|l|l|l|l|l|}
\hline$k$ & $v$ & $\sqrt{\mathfrak{c}_{4}}$ & $k$ & $v$ & $\sqrt{\mathfrak{c}_{4}}$ \\
\hline 0.001 & 0.870495 & 0.923901 & 1 & 0.873217 & 0.925689 \\
0.01 & 0.870519 & 0.923914 & 2 & 0.873242 & 0.925707 \\
0.05 & 0.870979 & 0.92419 & 3 & 0.873246 & 0.92571 \\
0.1 & 0.871755 & 0.924683 & 4 & 0.87324783 & 0.925711 \\
0.2 & 0.872616 & 0.925264 & 5 & 0.87324857 & 0.92571154 \\
0.3 & 0.872926 & 0.925481 & 6 & 0.87324898 & 0.92571183 \\
0.4 & 0.873058 & 0.925575 & 7 & 0.87324922 & 0.92571201 \\
0.5 & 0.873124 & 0.925622 & 8 & 0.87324968 & 0.92571234 \\
0.6 & 0.873161 & 0.925649 & 9 & 0.87324949 & 0.92571220 \\
0.7 & 0.873184 & 0.925665 & 10 & 0.87324957 & 0.92571226 \\
0.8 & 0.873199 & 0.925676 & 50 & 0.87324988 & 0.92571249 \\
0.9 & 0.87321 & 0.925684 & $10^{5} \geq k \geq 10^{2}$ & 0.87324990 & 0.92571249 \\
\hline
\end{tabular}

We can observe that for $k$ greater than 4 the ratio $\frac{v}{\sqrt{\mathfrak{c}_{4}}}$ is stabilized at a value around 0.943327. For large values of the wave number we will have the largest possible wave speed and thus the largest frequency.

In the Section 4, we have proved that in general we have at least one solution of the equation (4.7). But from the Figure 1, we can see that for the materials considered by Gauthier (1982), we have only one solution of this equation. So, the speed is uniquely determined.

The Figures 2, 3 and 4 provide the behavior of the displacement and the microrotation fields. Let us remark that we have maximum (minimum) values of the microrotation component in the neighborhood of minimum (maximum) values of the macrodisplacement component $u_{2}$.

A significant attenuation of the amplitude of the surface wave with respect to the deepness in the direction of the $x_{2}$ can be observed. If we look to the behaviour of the components $u_{1}, u_{2}$ and $\varphi$, from the above figures we can see that $u_{1}$ has a rapid decay with depth while the decays of $u_{2}$ of $\varphi$ are milder.

In the Figures 5, 6 and 7 we represent the behavior of the traction and of the couple traction.

A minimum of the couple traction occurs around a maximum value of the component $S_{21}$ of the stress tensor and vice versa. A similar decaying effect with respect to the deepness $x_{2}$, like that for the displacement and microrotation components, can be observed for the stress and couple stress components. 


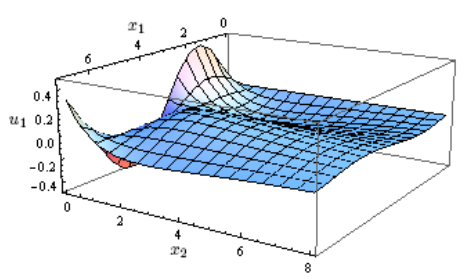

2: The component $u_{1}$ of the displacement vector for $k=1$ and the time $t=10$, on $\left(x_{1}, x_{2}\right) \in[0,7) \times[0,8)$.

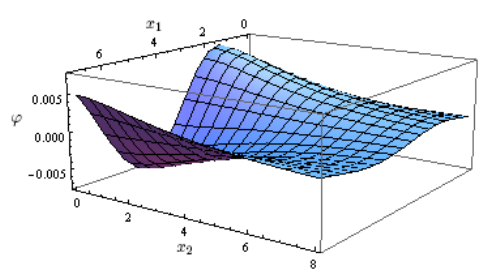

4: The component $\varphi$ of the microrotation vector for $k=1$ and the time $t=10$, on $\left(x_{1}, x_{2}\right) \in[0,7) \times[0,8)$.

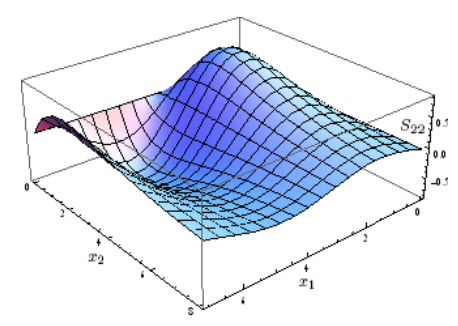

6: $S_{22}$ for $k=1$ and the time $t=10$, on $\left(x_{1}, x_{2}\right) \in[0,7) \times[0,8)$.

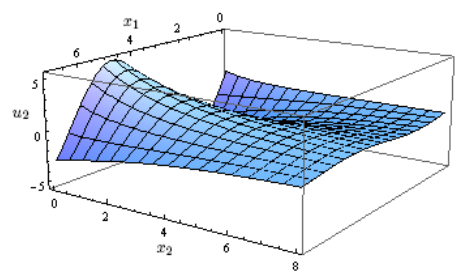

3: The component $u_{2}$ of the displacement vector for $k=1$ and the time $t=10$, on $\left(x_{1}, x_{2}\right) \in[0,7) \times[0,8)$.

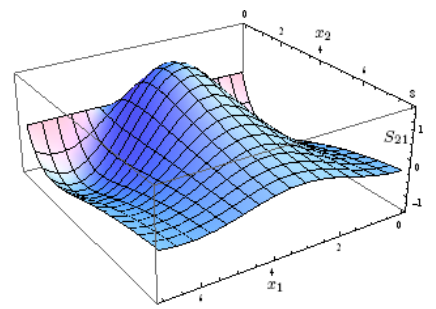

5: $S_{21}$ for $k=1$ and the time $t=10$, on $\left(x_{1}, x_{2}\right) \in[0,7) \times[0,8)$.

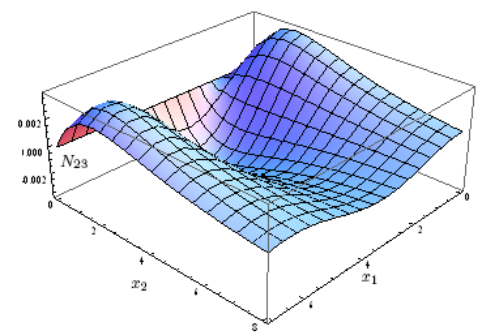

7: $N_{23}$ for $k=1$ and the time $t=10$, on $\left(x_{1}, x_{2}\right) \in[0,7) \times[0,8)$.

\section{References}

Achenbach, J.D., 1973. Wave Propagation in Elastic Solids. North-Holland Publishing Company, Amsterdam.

Chiriţă, S., Ghiba, I.D., 2010. Inhomogeneous plane waves in elastic materials with voids. Wave Motion 47, 333-342.

Cosserat, E., Cosserat, F., 1909. Théorie des corps déformables. A. Hermam et Fils, Paris.

Destrade, M., 2007. Seismic Rayleigh waves on an exponentially graded, orthotropic halfspace. Proc. R. Soc. A 463, 495-502.

Eringen, A.C., Suhubi, E.S., 1964. Nonlinear theory of simple microelastic solids. Int. J. Engng. Sci. 2, 189-203. 
Eringen, A.C., Suhubi, E.S., 1964. Nonlinear theory of simple microelastic solids II. Int. J. Engng. Sci. 2, 389-404.

Eringen, A.C., 1966. Linear theory of micropolar elasticity. J. Math. Mech. 15, 909-924.

Eringen, A.C., 1999. Microcontinuum Field Theories. Springer, New York.

Erofeyev, V.I., 2003. Wave processes in solids with microstructure. World Scientific, New Jersey.

Gauthier, R.D, Jahsman, W.E., 1975. A quest for micropolar elastic constants. J. Appl. Mech 42, 369-374.

Gauthier, R.D., 1982. Experimental investigations of micropolar media. in: Brulin, O, Hsieb, R.K.T. (Eds.), Mechanics of Micro-Polar Media. World Scientific, Singapore, pp. 395-463.

Georgiadis, H.G., Velgaki, E.G., 2003. High-frequency Rayleigh waves in materials with micro-structure and couple-stress effects. International Journal of Solids and Structures 40, 2501-2520.

Georgiadis, H.G., Vardoulakis, I., Velgaki, E.G., 2004. Dispersive Rayleigh-waves propagation in microstructured solids characterized by dipol gradient elasticity. J. Elasticity $74,17-45$.

Hayes, M., Rivlin, S.R., 1962. A note on the secular equation for Rayleigh waves. ZAMP $13,80-83$.

Jeffreys, H., 1952. The Earth, Cambridge University Press.

Ieşan, D., 2004. Thermoelastic Models of Continua. Kluwer Academic Publishers, London.

Koebke, R.H., Weitsman, Y., 1971. Surface wave propagation over an elastic Cosserat half-space. J. Acoust. Soc. Am. 50, 875-884.

Kulesh, M.A., Matveenko, V.P., Shardakov, I.N., 2005. On the propagation of elastic surface waves in the Cosserat medium. Doklady Physics 50, 601-604.

Kulesh, M.A., Matveenko, V.P., Shardakov, I.N., 2006. Propagation of surface elastic waves in the Cosserat medium. Acoustical Physics 52, 186-193.

Li, X.-F.,2006. On approximate analytic expressions for the velocity of Rayleigh waves. Wave Motion 44, 120-127.

Malischewsky, P.G., 2000. Comment to "A new formula for velocity of Rayleigh waves" by D. Nkemzi [Wave Motion 26 (1997) 199-205]. Wave Motion 31, 93-96.

Mindlin, R.D., 1963. Influence of couple-stresses on stress-concentrations. Exper. Mech. 3, 1-17. 
Mindlin, R.D., 1964. Microstructure in linear elasticity. Arch. Rational Mech. Anal. 16, $51-78$.

Nkemzi, D., 1997. A new formula for the velocity of Rayleigh waves. Wave Motion 26, 199-205.

Nkemzi, D.,2008. A simple and explicit algebraic expression for the Rayleigh wave velocity. Mechanics Research Communications 35, 201-205.

Nowacki, W., 1986. Theory of Asymmetric Elasticity. Pergamon Press, Oxford.

Rahman, M., Barber, J.R., 1995. Exact expression for the roots of the secular equation for Rayleigh waves. ASME J. Appl. Mech. 62, 250-252.

Lord Rayleigh, 1885. On waves propagated along the plane surface of an elastic solid. Proc. London Math. Soc. 17, 4-11.

Stroh, A.N., 1962. Steady state problems in anisotropic elasticity. J. Math. Phys. 41, $77-103$.

Ting, T.C.T., 2011. Surface waves in an exponentially graded, general anisotropic elastic material under the influence of gravity. Wave Motion 48, 335-344.

Ting, T.C.T., 2011. Secular equations for Rayleigh and Stoneley waves in exponentially graded elastic materials of general anisotropy under the influence of gravity. J. Elasticity DOI: $10.1007 /$ s10659-011-9314-9.

Toupin, R.A., 1962. Elastic materials with couple-stresses. Arch. Rat. Mech. Anal. 11, 385-414.

Truesdell, C., Noll, W., 1965. The Nonlinear Field Theories of Mechanics. in: Flugge S. (Ed.), Handbuch de Physik, vol III/3. Springer-Verlang, Berlin.

Vinh, P.C., Ogden, R.W., 2004. On formulas for the Rayleigh wave speed. Wave Motion 39, 191-197.

Vinh, P.C., Malischewsky, P.G., 2007. An approach for obtaining approximate formulas for the Rayleigh wave velocity. Wave Motion 44, 549-562.

Vinh, P.C., Malischewsky, P.G., 2008. Improved approximations of the Rayleigh wave velocity. Journal Of Thermoplastic Composite Materials 21, 337-352. 